\title{
DE LA PRESENCIALIDAD A LA DISTANCIA: MODELOS PARA LA INCORPORACIÓN DEL E-LEARNING EN LA UNIVERSIDAD
}

\author{
Dr. Jorge Grünberg* \\ Dr. Alejandro Armellini**
}

\begin{abstract}
Este artículo discute un marco de referencia para la conceptualización y evaluación de modelos de $e$ learning en educación superior.

Las modalidades "presencial" y "a distancia" han sido concebidas históricamente como los modelos educativos por excelencia conceptualizados como mutuamente contrapuestos. Sin embargo, las tecnologías de e-learning, posibilitan nuevos modelos educativos que combinan la presencialidad y la distancia de distintas maneras.

Sobre la base de la experiencia de la Universidad ORT Uruguay, planteamos un continuo a lo largo del cual aparecen modelos educativos en los que el e-learning y el aula presencial se complementan para lograr distintos objetivos. Las dimensiones pedagógicas, tecnológicas y económicas de estos modelos son abordadas en este artículo.

Las nuevas tecnologías de e-learning han atraído la atención de las instituciones educativas durante los últimos años. E-learning es definido como "el uso de tecnologías de Internet para brindar soluciones que mejoren el conocimiento y las prestaciones" en procesos educativos (Rosenberg, 2001: 28).

Desde escuelas técnicas a grandes universidades se han interesado en utilizar estas nuevas tecnologías para enseñar a través de barreras físicas y temporales sin necesidad de costosas inversiones en infraestructura física. Aunque algunos proyectos de e-learning han sido discontinuados en los últimos tiempos, en algunos casos luego de incurrir en grandes pérdidas, los analistas sugieren que esto puede ser parte de un proceso de consolidación en la industria y no una declinación de la misma (Adkins, 2002).

En la Universidad ORT utilizamos e-learning desde 1999. A partir de esta experiencia, este artículo ofrece un marco de referencia para la conceptualización y evaluación de modelos de e-learning en educación superior.
\end{abstract}

\section{Dimensiones del e-learning}

Cada modelo de e-learning implica opciones en diferentes dimensiones: tecnológica, económica y pedagógica.

Las decisiones tecnológicas suelen ser las que concentran mayor atención en las discusiones sobre $e$ learning y están, en general, fuertemente influenciadas por tecnólogos. El riesgo es que una estrategia dominada por el "pensamiento tecnológico" puede llevar a decisiones carentes de consideraciones pedagógicas o financieras. Un ejemplo clásico de pensamiento tecnológico (también llamado "pensamiento orientado por la tecnología") es asumir que si la tecnología está disponible, entonces alumnos y docentes van a incorporarla inexorablemente (Bates, 1995).

Los aspectos económicos suelen ser subestimados durante las etapas iniciales de los proyectos de $e$ learning. Es habitual que las instituciones educativas comiencen con acciones puntuales y de bajo riesgo, lideradas en forma individual por unos pocos docentes altamente motivados. Esto es usual en los procesos de difusión de innovaciones (Rogers, 1995; Klopfenstein, 1997). 
Sin embargo, para que el e-learning tenga un impacto real en la institución, es preciso involucrar números relativamente altos de profesores y cursos. A medida que el uso del e-learning se expande y sistematiza dentro de una institución, la planificación financiera se vuelve más importante para garantizar la sostenibilidad. Existe relativamente poca investigación relevante sobre estas cuestiones. Como consecuencia, los estudios económicos tienden a trasladar premisas desde la educación presencial tradicional al e-learning de modo acrítico, al igual que los primeros productores cinematográficos utilizaban las normas de escena del teatro en sus producciones. Las normas y presunciones asociadas al entorno presencial no son necesariamente aplicables al e-learning y en algunos casos pueden llevar a escenarios poco realistas (véase, por ejemplo, Davies y Quick, 2001, Smith et al, 2001; Bissell, 2002; Carnevale, 2002).

La pedagogía en la educación a distancia a través de Internet ha sido objeto de diversas investigaciones en los últimos años (Salmon, 2000; Palloff y Pratt, 2001). La discusión de esta literatura excede los propósitos de este trabajo. Sin embargo, es importante notar que en el e-learning las estrategias de intervención que el docente despliega tienden a depender de los recursos tecnológicos en mayor medida que en la educación presencial.

El e-learning es un campo complejo y cambiante, donde el liderazgo no puede ser provisto por tecnólogos, administradores o docentes de manera separada. Las decisiones de carácter estratégico deben ser tomadas a nivel institucional para asegurar que la innovación trascienda el esfuerzo individual aislado de docentes. Nuestra experiencia sugiere que un enfoque holístico, multidisciplinario y abarcador de las dimensiones mencionadas es necesario para concebir, lanzar y sostener aplicaciones de e-learning exitosas.

Un análisis estratégico sobrio y realista debe preceder decisiones tácticas y operativas sobre tecnología, economía o pedagogía. Este análisis consiste en una revisión sistemática de tres aspectos: a) los servicios educativos ofrecidos por la institución; b) aquellos servicios educativos existentes que la institución pretende mejorar o servicios nuevos que pretende lanzar; y c) de qué modo las tecnologías de e-learning pueden constituir medios efectivos en términos tecnológicos, económicos y pedagógicos para alcanzar los objetivos de b), contando con a) como punto de partida.

\section{Nuevos modelos educativos y e-learning}

La literatura muestra una concepción "binaria" de los procesos educativos en donde los estudiantes participan de manera totalmente "presencial" o totalmente "a distancia". Esta dicotomía ha sido objeto de críticas (por ejemplo, Weigel, 2000; Cronin, 2002).

En este artículo argumentamos que las tecnologías de e-learning permiten diseñar nuevos modelos educativos en donde la presencialidad y la distancia se combinan de distintas maneras. Estos nuevos modelos educativos pueden ser ordenados a lo largo de un continuo entre la presencialidad y la distancia "pura". Las opciones de carácter tecnológico, económico y pedagógico varían en las diferentes secciones de este continuo.

Los extremos del continuo están dados por las formas "puras" de presencialidad y educación a distancia (ver figura 1). En la Universidad ORT se dictan cursos a distancia "pura" a través de Internet desde 2000, cuando el Diploma en Educación, postgrado de un año de duración, dirigido a docentes en actividad, fue ofrecido por primera vez (Grünberg, 2001). Desde 2002, un segundo postgrado está siendo ofrecido con esta modalidad: el Diploma en Planificación y Gestión Educativa. 


\begin{tabular}{|l|l|}
\hline Presencialidad & Distancia \\
\cline { 2 - 2 } "pura" & "pura" \\
\hline
\end{tabular}

Figura 1

Ambas experiencias se posicionan en el extremo derecho del continuo. Este modelo apunta a resolver el acceso a cursos, disminuyendo la necesidad de proximidad y simultaneidad que demandan los cursos presenciales (para una discusión más extensa de los conceptos de proximidad y simultaneidad, ver Grünberg, 2001).

Un modelo educativo ubicado entre los dos extremos mencionados es la "webización" de cursos presenciales, es decir el uso de e-learning para apoyar, enriquecer o complementar cursos presenciales existentes (Bonk y Dennen, 1999). Este modelo (ver figura 2) difiere de los modelos a distancia "puros" en cuanto a sus objetivos y requerimientos para su implementación. La "webización"de cursos presenciales no apunta a enseñar los mismos cursos a alumnos distantes sino a mejorar los cursos a los alumnos actuales. Las tecnologías de e-learning facilitan la distribución y utilización de materiales educativos y bibliografía, permiten intercambios entre pares, apoyan actividades de colaboración, ofrecen oportunidades para la consulta a expertos y expanden las posibilidades de intercambio con el docente (Bonk y Dennen, 1999; Instructional Technology Resource Center, 2001).

La "webización" de cursos presenciales en la Universidad ORT Uruguay supera, a la fecha de elaboración de este artículo, los 180 docentes en 250 cursos. De acuerdo con nuestras investigaciones, uno de los beneficios más valorados de este modelo de uso de e-learning es la posibilidad de realizar discusiones estructuradas que trascienden los límites rígidos de tiempo y espacio de los encuentros en el aula. Esto incrementa la participación del alumno, propiciando una cobertura más profunda del programa y la capacidad de elaborar y fundamentar argumentos propios (Armellini y Gregori, 2001, Instructional Technology Resource Center, 2001). Desde el punto de vista institucional, la "webización" de cursos puede constituir un diferenciador estratégico entre una institución y sus competidores, así como un factor de motivación y retención de estudiantes.

\begin{tabular}{|l|l|l|}
\hline Presencialidad & "Webización" de & $\begin{array}{l}\text { Distancia } \\
\text { "pura" }\end{array}$ \\
\cline { 2 - 3 } & cursos presenciales & \\
\hline
\end{tabular}

Figura 2

Alejándonos un poco más del modelo presencial tradicional, encontramos un modelo utilizado actualmente por la Universidad ORT para la formación docente en servicio (ver figura 3). La mayor parte de los profesores de la Universidad ORT son especialistas en sus áreas académicas pero carecen de conocimiento sistemático sobre pedagogía o aprendizaje.

Todos los años ofrecemos cursos de formación docente en servicio para compensar esta carencia. Tradicionalmente, estas instancias han contado con escasa asistencia por conflictos de horarios y porque estos cursos resultan demasiado cortos para tener un impacto real en las prácticas de enseñanza. Sin embargo, compromisos dentro y fuera de la Universidad impiden a nuestros docentes asistir a cursos de mayor duración. 


\begin{tabular}{|l|l|l|l|}
\hline \multirow{2}{*}{$\begin{array}{l}\text { Presencialidad } \\
\text { "pura" }\end{array}$} & "Webización" de & $\begin{array}{l}\text { Cursos } \\
\text { cursos presenciales }\end{array}$ & $\begin{array}{l}\text { Distancia } \\
\text { "híbridos" }\end{array}$
\end{tabular}

Figura 3

El Certificado en Docencia Universitaria apunta a mejorar las prácticas de enseñanza y se ofreció por primera vez en el año. Se trata de un programa de ocho meses que combina instancias presenciales con componentes a distancia basados en Internet.

El Certificado incluye un número relativamente bajo de encuentros presenciales, combinados con períodos de intercambios electrónicos a distancia. Típicamente estas actividades no presenciales se extiendan por lapsos de tres a cinco semanas. Este enfoque ha demostrado ser atractivo para los docentes en cuanto a su profundidad y cobertura temática, siendo a la vez compatible con sus horarios.

Este enfoque es cualitativamente diferente del apoyo a cursos presenciales a través de Internet discutido anteriormente, no sólo en cuanto a la proporción de encuentros presenciales con respecto a la actividad a distancia, sino también en cuanto a objetivos y estrategias de intervención. En el caso de cursos presenciales apoyados por Internet, la columna vertebral del curso consiste en una secuencia de encuentros presenciales regulares y frecuentes, con "extensiones virtuales" facilitadas por la tecnología.

En el modelo "híbrido" del Certificado en Docencia Universitaria, los componentes presenciales y a distancia se complementan mutuamente tanto en el plano social como en el pedagógico. Las instancias presenciales esporádicas proveen la motivación y cohesión que permiten a los participantes sentirse parte de un grupo con metas comunes. Las discusiones electrónicas ofrecen un espacio extendido de reflexión y análisis de los temas abordados en el aula. Un número excesivo de instancias presenciales puede implicar un costo elevado para los participantes, mientras que demasiado pocas pueden resultar insuficientes para mantener la motivación y cohesión en el grupo (especialmente en programas voluntarios de formación continua).

Conceptualmente, los cuatro modelos presentados en la figura 3 pueden ser concebidos como mojones de un continuo y no como compartimientos discretos. A manera de ejemplo, los sistemas presenciales pueden hacer uso de tecnologías de e-learning para brindar servicios adicionales como trabajos remediales, habilidades generales (por ejemplo comunicación, expresión escrita o informática) o evaluación formativa. De este modo, los alumnos pueden recibir el apoyo o complemento que personalmente necesitan, cuando lo necesitan, sin competir por el tiempo presencial de los cursos regulares de sus carreras.

Análogamente, los modelos a distancia pueden beneficiarse de encuentros presenciales para orientación, consulta o motivación. Las instituciones educativas locales pueden competir con los actores globales en el mercado del e-learning justamente por su habilidad de ofrecer el componente presencial humano y local, sin el cual los cursos dictados totalmente a distancia a través de Internet, pueden tornarse, social y psicológicamente intolerables. 


\section{Conclusión: las tecnologías de e-learning como agentes de cambio}

El análisis y la evaluación del continuo de modelos educativos que pueden instrumentarse utilizando tecnologías de e-learning, contribuyen a orientar innovaciones educativas en términos tecnológicos, económicos y pedagógicos.

El impacto de las tecnologías de e-learning no se limita a la implementación de nuevas formas de educación a distancia, sino a posibilitar el desarrollo de un amplio espectro de nuevos modelos educativos que combinan de distintas maneras la presencialidad y la distancia.

Puede pensarse que el impacto de las tecnologías de e-learning en los sistemas educativos sea creciente con el tiempo. Herramientas informáticas para el desarrollo de cursos a distancia, amigables y de bajo costo, continuarán desarrollándose e incorporándose a las prácticas educativas. Los docentes avanzarán en sus curvas de aprendizaje, incorporando y perfeccionando estrategias de enseñanza que incluirán componentes de e-learning.

Todo esto se traducirá en nuevas oportunidades de acceso a servicios educativos y en un mayor grado de flexibilidad para los estudiantes que, progresivamente, podrán armar sus propios modelos de participación, eligiendo el mix más conveniente de presencialidad y distancia de acuerdo con sus estilos de aprendizaje, requerimientos y prioridades personales y profesionales.

El fuerte desarrollo de tecnologías de e-learning puede demostrar su importancia en el largo plazo, no solamente como plataforma de crecimiento para las instituciones educativas, sino por su potencial para catalizar innovaciones en sistemas educativos existentes, los cuales han estado organizados esencialmente de la misma manera por cientos de años.

\section{Bibliografía}

Adkins, S. 2002. Market Analysis of the 2002. U.S. E-Learning Industry Convergence, Consolidation and commoditization, Brandon-hall.com 2002 Market Analysis Series.

Armellini, A. \& Gregori, M. 2001. Criterios para la incorporación efectiva de Internet como apoyo a cursos presenciales. Informe de investigación. Montevideo: Universidad ORT Uruguay.

Bates, A. 1995. Technology, open learning and distance education. Londres: Routledge.

Bissell, C. 2002. Supporting student projects at a distance through ICT: the UK Open University approach. European Journal of Engineering Education, 27(1), 5-12.

Bonk, C. and Dennen, V. 1999. Teaching on the web: With a little help from my friends. Journal of Computing in Higher Education, 11(1), 3-28.

Carnevale, D. 2002. Online students don't fare as well as classroom counterparts, study finds. Chronicle of Higher Education, 48(27).

Cronin, B. 2002. Locus Classicus. Library Journal, 127(11), p 44.

Davies, T. \& Quick, D. 2001. Reducing distance through distance learning: the community college leadership doctoral program at Colorado State University. Community College Journal of Research \& Practice, 25(8), 607-620.

Grünberg, J. (2001) Using the Internet in the delivery of educational services. Concepts and practical implementation. Trabajo presentado en el Foro de Directores de World ORT, Oxford, Marzo de 2001.

Instructional Technology Resource Center 2001. (online), Idaho State University. www.isu.edu/itrc, accedido el 01/08/2002. 
Klopfenstein, B. 1997. (online) Diffusion of innovations on the web. www.bgsu.edu/departments/tcom/ diffusion.html, accedido el 10/08/2002.

Palloff, R. \& Pratt, K. 2001 Lessons from the cyberspace classroom. Chichester: John Wiley and Sons Ltd.

Rogers, E. 1995. Diffusion of innovations (4ta edición). Nueva York: The Free Press.

Rosenberg, M. 2001. E-Learning. Nueva York: McGraw-Hill.

Salmon, G. 2000 E-Moderating: The key to teaching and learning online. Oxford: OUP.

Smith, G.; Ferguson, D. \& Caris, M. 2001 Online vs face-to-face. T H E Journal, 28(9), 18-24.

Weigel, V. 2000 E-Learning and the tradeoff between richness and reach in higher education. Change, 33(5), 10-15.

*Ph.D. en Educación, Universidad de Oxford. Master en Computación, Universidad de Oxford. Ingeniero de Sistemas. Rector, Universidad ORT.

**Ph.D. en Tecnología Educativa, Universidad de Kent, Inglaterra. Master en Tecnología Educativa, Universidad de Manchester, Inglaterra. Learning Technologist, Unit for the Enhancement of Learning and Teaching, University of Kent at Canterbury.

Una versión en inglés de este artículo, titulada "E-learning: the strategy continuum", fue presentada en el Foro Mundial de Directores Nacionales de ORT en Nueva York, en junio de 2002. 